\title{
Effective use of computed tomography to distinguish an insurmountable tortuosity of the common iliac artery during the trans-femoral approach
}

\begin{abstract}
An 81-year-old-man presenting with syncope as main complaint showed significantly elevated cardiac enzymes the following day, and was referred for percutaneous coronary intervention (PCI) via the right femoral artery. Significant tortuosity of the right common iliac artery with severe calcification hampered the catheter manipulation for PCI. This could have been predicted by calculating the median luminal centerline-straight line ratio from 3D computed tomography before the PCI procedure. Evaluation of the tortuosity of the common iliac and femoral arteries should precede all PCI procedures using the transfemoral approach
\end{abstract}

Keywords: tortuosity, 3D-computed tomography, percutaneous coronary intervention

\author{
Volume I Issue 6 - 2014 \\ Hiroki Kitakata,Teruo Okabe, Yasuo Kurita, \\ Narutaka Ohashi,Yukiko Karube, Satoshi \\ Ogawa \\ Cardiovascular Center, Mita Hospital, Japan
}

\begin{abstract}
Correspondence: Teruo Okabe, Cardiovascular Center, Mita Hospital , I-4-3 Mita, Minato-ku, I08-8329, Tokyo, Japan, Tel 03-
\end{abstract} 345I-8I2I,Email tmsbI05@iuhw.ac.jp

Received: September 04, 2014 | Published: November 27 2014
Abbreviations: PCI, percutaneous coronary intervention; AMI, Acute Myocardial Infarction; CT, computed tomography; ACS, acute coronary syndrome; LAD, left anterior descending; RCA, artery right coronary artery

\section{Introduction}

Tortuosity of the common iliac or femoral artery has come to be regarded as one of the important factors for successful percutaneous intervention via a femoral approach. ${ }^{1-4}$ Even though earlier reports referred to cases of endovascular abdominal aortic stent graft deployment in the tortuosity of those vessels, the same fundamental technical difficulties might be encountered. Although, the trans-radial approach is gaining in popularity,,${ }^{5,6}$ a large number of percutaneous coronary intervention (PCI) procedures are still performed via femoral artery access, especially in emergent cases. ${ }^{5,7}$ or in patients undergoing hemodialysis. Excessive tortuosity of the common iliac or femoral artery may impede the manipulation of the guiding catheter and guide wire. Prior to the procedure, an assessment of whether tortuosity could be a serious impediment to PCI is very difficult to achieve in a non invasive manner. Here, we report the case of a patient with an acute myocardial infarction (AMI) whose severely tortuous common iliac artery seriously impeded the PCI procedure.

\section{Case report}

An 81-year old man had been attending a physician at our hospital for several years, because of type II diabetes mellitus, hypertension, and chronic kidney disease. On one regular visit to the doctor, sudden syncope and collapse within the hospital required him to be admitted to the department of internal medicine. The patient recovered consciousness several minutes later and no apparent neurological abnormality could be detected. Cardiovascular examination identified no significant heart murmur. Blood tests showed no evidence of myocardial enzyme leakage, electrolyte disturbance, inflammation, or significant anemia that might be associated with syncope, except for the fact that the troponin I level was just above the upper normal limit $(0.11 \mathrm{ng} / \mathrm{mL})$. The electrocardiogram also showed no specific changes suggestive of an acute coronary syndrome (ACS). The chest X-ray depicted a status of mild congestion, and echocardiography showed signs of mild hypokinesis of the posterior wall. Brain computed tomography (CT) showed no evidence of fresh infarction or hemorrhage at this point. Enhanced CT from chest to pelvis did not indicate any evidence of aortic dissection or aneurysmal rupture, but there was significant tortuosity of the right common iliac artery and the abdominal aorta below the renal arteries, with severe calcification (Figure 1). The next day's blood test, however, showed significant increases in the levels of CK (2823IU/L), AST (433IU/L), LDH $(1231 \mathrm{IU} / \mathrm{L})$, and $\mathrm{WBC}(132.1 \times 100 / \mu \mathrm{L})$, signifying the development of acute myocardial infarction. The coronary angiography (CAG) was performed via the right femoral artery, using $5 \mathrm{~F}$ diagnostic catheters, JL5.0 and JR4, and showed a 90\% stenosis in the left anterior descending artery (LAD)\#6-7, with TIMI grade III flow, and a total occlusion in the right coronary artery (RCA) $\# 2$, with a relatively steep angulations (Figure 2). Accordingly, PCI was performed, treating the RCA occlusion as a culprit on the same day. Since the patient suffered from chronic kidney disease, as indicated by the high creatinine value $(1.85 \mathrm{mg} / \mathrm{dL})$ and low estimated glomerular filtration rate $(21.5 \mathrm{~mL} /$ $\min / 1.73 \mathrm{~m}^{2}$ ), we selected the right femoral artery for access, in order to preserve the radial arteries for shunts in the case of hemodialysis. Although the left femoral artery was also considered as a candidate for access, the weak pulsation of both left popliteal and dorsal arteries kept us from choosing it. Even though the enhanced CT indicated this case as a high complicated case about its vessel tortuosity, we continued the procedure with disregard for this information at this point. A 7F super-long sheath was inserted in order to elongate the tortuosity of the common iliac artery, but the sheath became kinked at the tortuous point. Consequently, the guiding catheters could not be manipulated effectively, because of the tight constriction of each catheter by the sheath. We sized down the catheter from $7 \mathrm{~F}$ to $5 \mathrm{~F}(7 \mathrm{~F}$ JR4.0, SAL0.75, and AL1.0, 6F AL1.0, and 5F JR4.0) so as to allow more space, but this was unsuccessful. Finally, we decided to change the access site to the right brachial artery with a $6 \mathrm{~F}$ sheath, and we selected a $6 \mathrm{~F}$ EBU3.5 as a guiding catheter. As we carefully manipulated the catheter, in which the Radiocast ${ }^{\circledR}$ Guide wire (TERUMO, Tokyo, 
Japan) was still inserted, the tip of the catheter passed into the RCA, though not very deeply. The ULTIMATEbros3 ${ }^{\circledR}$ guidewire (ASAHI INTECC, Aichi, Japan) was successfully advanced to \#4AV with support from a microcatheter, (Finecross ${ }^{\circledR}$ MG, TERUMO, Tokyo, Japan), after an attempt to cross the lesion witha floppy guidewire had failed (Joker ${ }^{\circledR}$, Japan Lifeline, Tokyo, Japan) (Figure 3). The lesion was then dilated using a $1.2 \mathrm{~mm}$ semi-compliant balloon $(T E N K U \AA$, ST. JUDE MEDICAL, St Paul, MN, USA) followed by a $2.5 \mathrm{~mm}$ balloon (Lacrosse LAXA ${ }^{\circledR}$, GOODMAN, Aichi, Japan). At this point, we established that there was recovery flow of TIMI II, with huge amounts of floating thrombus. Unfortunately, the aspiration was completely unsuccessful, so we decided to compress the thrombus as far as possible using a large-sized balloon. From the occlusion site to distal portion\#3, the vessel was entirely dilated by a $3.5 \mathrm{~mm}$ semicompliant balloon (Sprinter ${ }^{\circledR}$ legend RX, Medtronic, Minneapolis, $\mathrm{MN}$, USA), followed by a $5.0 \mathrm{~mm}$ non-compliant balloon (NC Quantum Apex ${ }^{\circledR}$, Boston Scientific, Natick, MA, USA). Finally, we determined that it would be better to terminate the procedure at this point without any stent deployment, because of the abundance of fresh thrombus throughout the RCA (Figure 4).
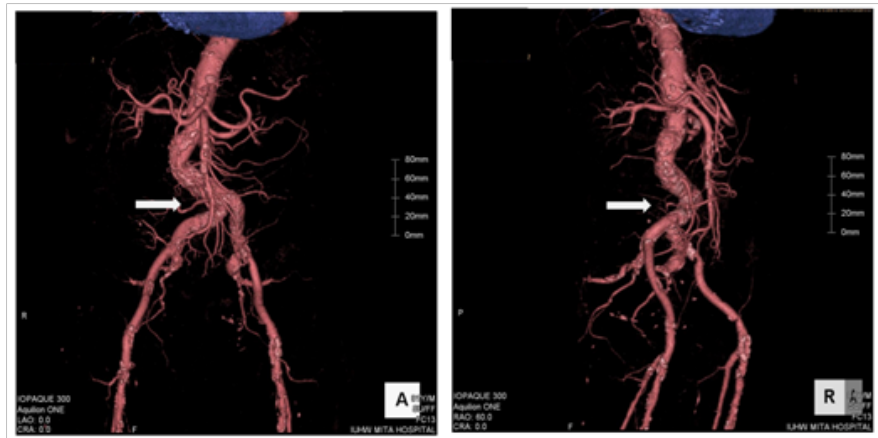

Figure I Three-dimensional image from enhanced computed tomography. The white arrows indicate significant tortuosity of the right common iliac artery. Left: antero-posterior projection. Right: right anterior-oblique projection.

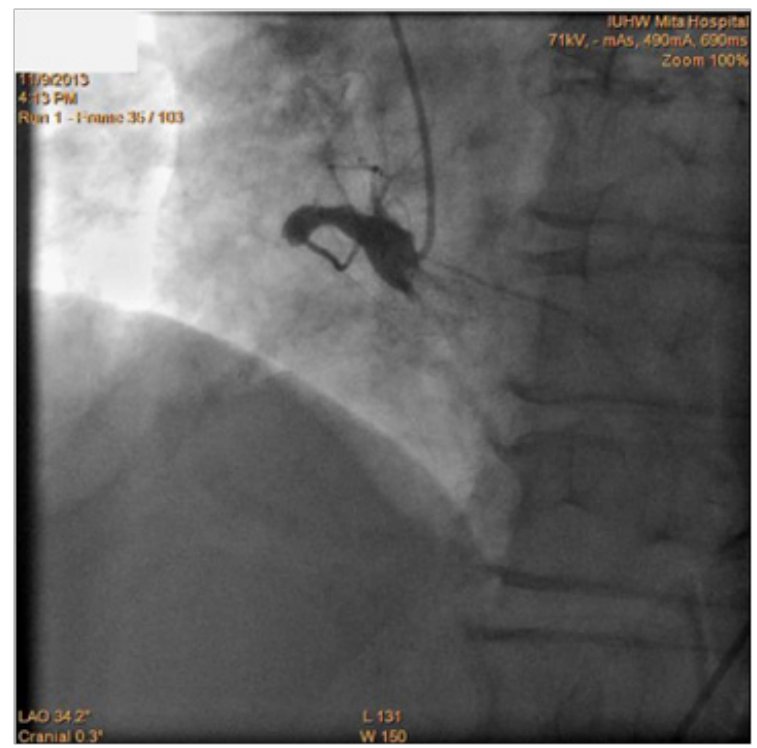

Figure2 Coronary angiography showing the right coronary artery before the percutaneous coronary intervention procedure.

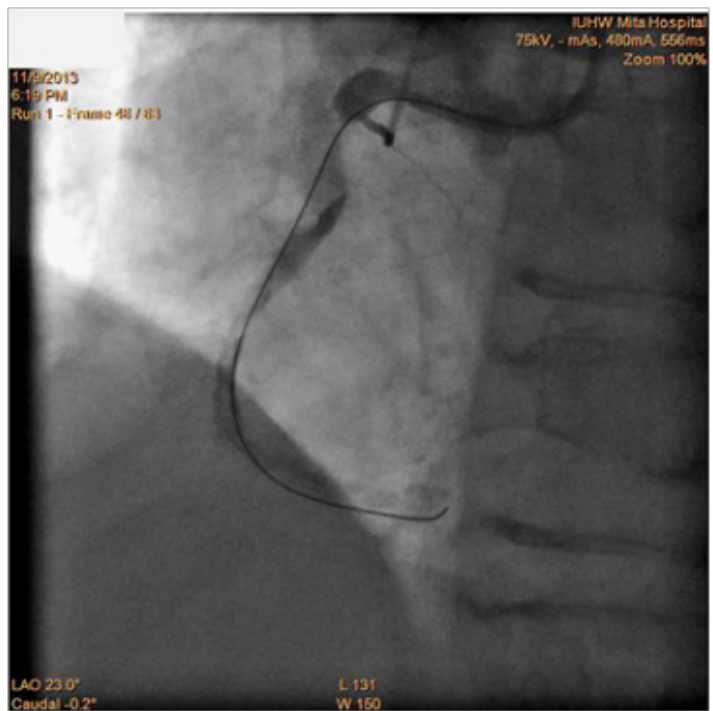

Figure 3 Successful passage of the ULTIMATEbros $3 \circledR$ guidewireinto the distal portion of the right coronary artery.

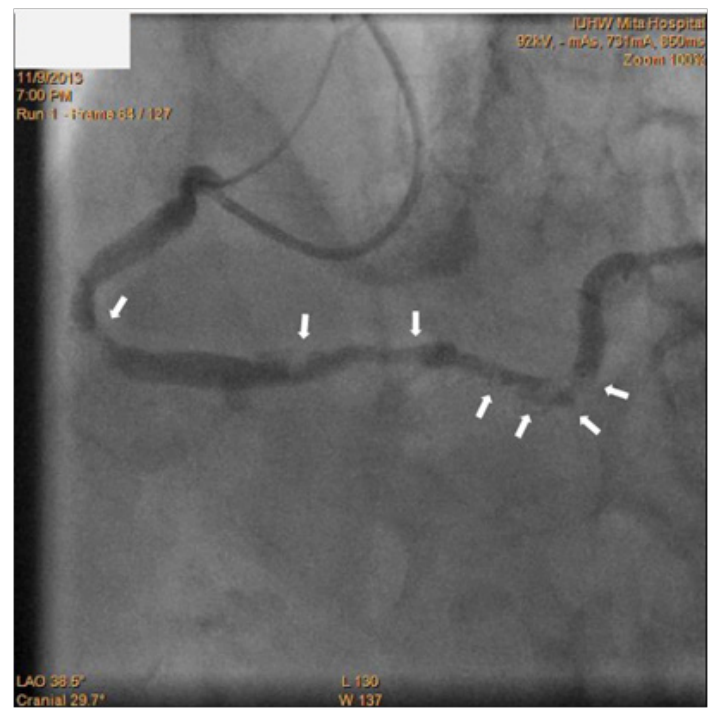

Figure 4 Final images after the percutaneous coronary intervention procedure, with an abundance of fresh thrombus throughout the right coronary artery. White arrows indicate thrombus.

\section{Discussion}

Tortuosity of the common iliac or femoral artery is an important factor for successful PCI viaa femoral approach. ${ }^{1-4}$ Several kinds of device have been invented in order to resolve this problem, such as ananti-kink sheath, or a thicker sheath with a wider lumen. These are based on the concept of straightening out the tortuous vessel as much as possible, or maintaining the inner lumen of the sheath while increasing its conformability to the vessel. Even so, a PCI procedure may result in failure if the sheath will not pass the tortuous vessel without kinking, or is unable to straighten the tortuosity.

Wolf et al. ${ }^{1}$ reported that the difference between the actual distance and the straight line distance of the vessel, defined as the ratio 
between a median luminal centerline and a straight line on CT images, might be closely associated with the rate of procedural complications, including sheath kink or inability to straighten tortuosity. Calcification at a tortuous site could also serve as an additional predictive factor for these technical failures. If we had recognized prospectively that this case had a relatively high tortuosity index (1.52) and a severe calcification at the tortuous site (Figure 5), we could have paid more attention to the selection of the approach site, including consideration of a trans-radial or trans-brachial approach before the start of the procedure. The evaluation of the 3D CT examination was performed on another day, after the procedure, since this was an emergent case. Moreover, the presence of chronic kidney disease biased us against using those alternative approaches rather thana femoral one. ${ }^{8,9}$ Eventually, we waste da range of catheters and incurreda long fluoroscopy time.

Of course, it is not practical for all patients in whom a trans-femoral PCI procedure is being scheduled to undergo an enhanced CT in order to evaluate the tortuosity of the common iliac or femoral artery before the procedure. The excess of dye and of radiation exposure prohibit such a strategy. However, a simple evaluation of the tortuosity of the vessel might be achieved by imaging with administration of small amounts of contrast via a short sheath just after its insertion. If a simple median luminal centerline to straight line ratio, measured in this way, were to be above a certain value, it might be better to seek alternative approaches in order to avoid struggling with tortuous vessels, even when there are other factors arguing against the selection of another site.

The course of the RCA in this patient was, incidentally, characterized by a relatively high take-off with steep angulations at the shoulder (Figure 2). We were afraid of the possibility that the tip of an AL1.0 catheter inserted via the trans-brachial route might penetrate deeply into the RCA, resulting in injury to the site of the culprit lesion. Thus, an EBU3.5 appeared to be a better choice, as it would not intrude so deeply but would fit well to the course of the RCA \#1 segment. The insufficiency of back-up power from this guiding catheter, however, required additional support from a guide wire. A stiffer guide wire with a micro-catheter, like the ULTIMATEbros $3{ }^{\circledR}$ plus Finecross ${ }^{\circledR}$ MG used in this case, was more effective than a floppy guide wire alone.

\section{Conclusion}

Tortuosity of the common iliac or femoral artery is an important factor for a successful PCI from a femoral approach. If an enhanced abdominal $\mathrm{CT}$ has been performed for some reason, the median luminal centerline to straight line ratio should be calculated before the procedure. If not, before starting any trans-femoral PCI procedure, imaging through a short sheath might be recommended, in order to check for the existence of a severely tortuous vessel that could impede or prevent the procedure.

\section{Acknowledgments}

None.

\section{Conflicts of interest}

Authors declare that there is no conflict of interest.

\section{References}

1. Wolf YG, Tillich M, Lee WA, et al. Impact of aortoiliac tortuosity on endovascular repair of abdominal aortic aneurysms:evaluation of 3D computer based assessment. J Vasc Surg. 2001;34(4):594-599.

2. Chuter TA, Reilly LM, Kerlan RK, et al. Endovascular repair of abdominal aortic aneurysm: getting out of trouble. Cardiovasc Surg.1998;6(3):232239.

3. Henretta JP, Karch LA, Hodgson KJ, et al. Special iliac artery considerations during aneurysm endografting. Am J Surg. 1999;178(3):212-218.

4. Lee AM, Rockman CB, Riles TS, et al. Report of a single institution experience using the EVT endovascular abdominal aortic aneurysm graft in 25 patients. Ann Vasc Surg. 1999;13(1):60-66.

5. Jolly SS, Yusui S, Cairns J, Niemela K, et al. Radial versus femoral access for coronary angiography and intervention in patients with acute coronary syndromes (RIVAL): a randomized parallel group, multicentre trial. Lancet. 2011;377(9775):1409-1420.

6. Secco GG, Marinucci L, Uguccioni L, et al. Transradial versus transfemoral approach for primary percutaneous coronary interventions in elderly patients. J Invasive Cardiol. 2013;25(5):254-256.

7. Mackram FE, Charanjit SR, Rajiv G, et al. Systematic use of transradial PCI in patients with ST-segment elevation myocardial infarction: a call to “arms”. JACC Cardiovasc Interv. 2013;6(11):1145-1148.

8. Zhenxian Yan, Yujie Zhou, Yingxin Zhao, et al. Impact of transradial coronary procedures on radial artery. Angiology. 2010;61(1):8-13.

9. Yonetsu T, Kakuta T, Lee T, et al. Assessment of acute injuries and chronic intimal thickening of the radial artery after transradial coronary intervention by optical coherence tomography. Eur Heart J. 2010;31(13):1608-1615. 